\title{
ON HOLONOMY COVERING SPACES
}

\author{
LOUIS AUSLANDER ${ }^{1}$
}

Introduction. In [1] L. Markus and the author introduced the concept of holonomy covering spaces for flat affinely connected manifolds or what are also called locally affine spaces. We also proved in [1] that the holonomy covering space of a complete $n$ dimensional locally affine space must be some $n$ dimensional cylinder, i.e., $T^{i} \times F^{n-i}, i=0, \cdots, n$, where $T^{i}$ denotes the locally affine $i$ dimensional torus and $E^{n-i}$ denotes the $n-i$ dimensional affine space. It is the purpose of this paper to prove that all these holonomy covering spaces are actually realized. To be more explicit, we shall construct $n$ locally affine connections $A_{i}^{n}, i=1, \cdots, n$, on the $n$ dimensional torus such that the holonomy covering space of $A_{i}^{n}$ is $T^{i} \times F^{n-i}$, $i=1, \cdots, n$.

1. General considerations. Let $E^{n}$ be the $n$ dimensional affine space and let $\Pi$ be a subgroup of the group of affine transformations of $E^{n}$. Let us assume that $\Pi$ acts on $E^{n}$ without fixed points and properly discontinuously, i.e., without accumulation points. We shall define two points $x$ and $y$ in $E^{n}$ to be equivalent if there exists a $g \in \Pi$ such that $g(x)=y$. Then the equivalence classes of $E^{n}$ with the induced topology form a manifold which we shall denote by $E^{n} / \Pi$. The class of such manifolds is precisely the class of complete locally affine spaces. If we let $E^{n}$ have $\left(x_{1}, \cdots, x_{n}\right)$ as coordinate systems, then the elements of $\Pi$ may be represented as $(n+1) \times(n+1)$ matrices whose last row consists of all zeros except in the $n+1$ entry where it is one. Let $g \in \Pi$. We may associate to $g$ the $n \times n$ matrix consisting of the first $n$ rows and columns of the matrix representation of $g$. Call this mapping $H$. Then it is an easy consequence of the discussion in [1] that $H(\mathrm{II})$ is precisely a matrix representation for the holonomy group of the space $E^{n} / \Pi$ with its induced affine connection. If $k$ (II) denotes the kernel of this homomorphism $H$, then $k(\Pi)$ is the fundamental group of the holonomy covering space of $E^{n} / \Pi$. Hence $k(\Pi)$ is a free abelian group on at most $n$ generators. Under the assumption that $k(\Pi)$ has $q$ generators, the holonomy covering space of $E^{n} / \Pi$ is $T^{q} \times E^{n-q}, q=1, \cdots, n$.

Hence to determine the desired affine connections $A_{i}^{n}, i=1, \cdots, n$,

Received by the editors July 29, 1955.

${ }^{1}$ During part of the time the author was writing this paper he was a National Science Foundation Post-Doctoral Fellow. 
we have to construct groups $\Pi_{i}^{n}, i=1, \cdots, n$, which have the following two properties:

1. $E^{n} / \Pi_{i}^{n} \approx T^{n}$.

2. $k\left(\Pi_{i}^{n}\right)$ has $n-i$ generators, $i=1, \cdots, n$.

2. Reduction of the problem. We shall now show that the knowledge of $\Pi_{1}^{2}, \Pi_{2}^{2}, \Pi_{3}^{3}$ is sufficient for constructing $\Pi_{i}^{n}, i=1, \cdots, n$.

CASE 1 . $i=1$ and $n \geqq 2$.

Let $\Pi_{1}^{2}(1)$ acting on $\left(x_{1}, \cdots, x_{n}\right)$ act as $\Pi_{1}^{2}$ on $x_{1}$ and $x_{2}$ and the identity mapping on the other coordinates. Let $P_{j}$ be the unit translation in the $j$ direction. Let $\Pi_{1}^{n}$ be the group generated by $\Pi_{1}^{2}(1)$ and $P_{j}, 3 \leqq j \leqq n$.

CASE 2 . $i$ even and $n \geqq 2$.

Let $2 \alpha=i$. We consider the affine transformation $\Pi_{2}^{2}(q)$, $q=1, \cdots, \alpha$, which is the identity transformation except on $x_{2 q-1}$, $x_{2 q}$ which it transforms as $\Pi_{2}^{2}$. Let $\Pi_{i}^{n}$ be the group generated by $\Pi_{2}^{2}(q), q=1, \cdots, \alpha$, and $P_{j}, i+1 \leqq j \leqq n$.

CASE 3. $i$ odd and $n \geqq 2$.

Let $i-3=2 \alpha$. Let $\Pi_{3}^{3}(i)$ be the identity transformation except on $x_{2 \alpha+1}, x_{2 \alpha+2}, x_{2 \alpha+3}$ which it transforms as $\Pi_{3}^{3}$. Let $\Pi_{\imath}^{n}$ be generated by $\Pi_{3}^{3}(i), \Pi_{2}^{2}(q), q=1, \cdots, \alpha$, and $P_{j}, i+1 \leqq j \leqq n$.

It is now a trivial matter to prove that $\Pi_{i}^{n}$ has the desired properties.

From the work of Kuiper we see that we must take $\Pi_{1}^{2}$ as the group generated by

$$
\begin{array}{lll}
x^{\prime}=x+y & \text { and } & x^{\prime}=x+1, \\
y^{\prime}=y+1 & y^{\prime}=y
\end{array}
$$

and $\Pi_{2}^{2}$ as the group generated by

$$
\begin{array}{lll}
x^{\prime}=x+y & \text { and } & x^{\prime}=x+q y+\lambda, \\
y^{\prime}=y+1 & y^{\prime}=y+q
\end{array}
$$

where $\lambda \neq q(q-1) / 2$ and $q$ is irrational.

3. On $\Pi_{3}^{3}$. Let $g_{1}, g_{2}, g_{3}$ be the transformations

$$
\begin{array}{lll}
x^{\prime}=x+y, & x^{\prime}=x+z, & x^{\prime}=x+q y+q z+k, \\
y^{\prime}=y+1, & y^{\prime}=y, & y^{\prime}=y+q, \\
z^{\prime}=z, & z^{\prime}=z+1, & z^{\prime}=z+q,
\end{array}
$$

respectively, where $0<q<1 / 8$ is irrational and $k \geqq 4$. Let $\Pi_{3}^{3}$ be the group generated by $g_{1}, g_{2}$, and $g_{3}$. It is a perfectly straightforward mat- 
ter to prove that $\Pi_{3}^{3}$ is abelian and that the general term $g_{1}^{n} g_{2}^{m} g_{3}^{s}$ of $\Pi_{3}^{3}$ is given by

$$
\begin{aligned}
& x^{\prime}=x+(n+s q) y+(m+s q) z+\lambda \\
& y^{\prime}=y+n+s q \\
& z^{\prime}=z+m+s q
\end{aligned}
$$

where

$$
\lambda=s k+n s q+m s q+2 q^{2} f(s)+f(n)+f(m)
$$

and

$$
f(n)= \begin{cases}\sum_{i=1}^{n-1} i, & n>0 \\ \sum_{i=1}^{-n} i, & n<0\end{cases}
$$

This can be simplified by noting that $f(n)=\left(n^{2}-n\right) / 2$ for all $n$. Hence

$$
\lambda=s k+q^{2}\left(s^{2}-s\right)+1 / 2\left(m^{2}-m\right)+1 / 2\left(n^{2}-n\right)+n s q+m s q
$$

or

(2) $\lambda=1 / 2(n+s q)^{2}+1 / 2(m+s q)^{2}+s\left(k-q^{2}\right)-1 / 2 n-1 / 2 m$.

LEMMA $1 . \Pi_{3}^{3}$ acts on $E^{3}$ without fixed points.

Proof. Let us assume there is a fixed point. Then by equation (1) we must have $n+s q=0$. But this is impossible since $q$ is irrational.

LEMMA 2. $\Pi_{3}^{3}$ acts properly discontinuously on $E^{3}$.

Proof. Let us assume that this is false. Then there exist $\left(n_{i}, m_{i}, s_{i}\right)$, $i=1,2, \cdots$, and a point $\left(x_{0}, y_{0}, z_{0}\right)$ such that $g_{1}^{n_{i}} g_{2}^{m_{i}} g_{3}^{s_{i}}\left(x_{0}, y_{0}, z_{0}\right)$ is a Cauchy sequence in $E^{n}$. Hence by equation (1), $n_{i}+s_{i} q$ and $m_{i}+s_{i} q$, $i=1,2, \cdots$, must be Cauchy sequences. But since $q$ is irrational this implies that $\lim _{i \rightarrow \infty}\left|n_{i}\right|=\infty, \lim _{i \rightarrow \infty}\left|m_{i}\right|=\infty$, and $\lim _{i \rightarrow \infty}\left|s_{i}\right|=\infty$. Further $s_{i}$ and $n_{i}$ must have opposite signs as must $s_{i}$ and $m_{i}$. But by equation 2 we see that if $x_{i}$ is the $x$ coordinate of $g_{1}^{n_{i}} g_{2}^{m_{i}} g_{3}^{s_{i}}\left(x_{0}, y_{0}, z_{0}\right)$, then $\lim _{i \rightarrow \infty}\left|x_{i}\right|=\infty$. This contradiction completes the proof of this lemma.

We shall use a bracket about a set of points in $E^{3}$ to denote the point set determined by the closed simplex which has these points as vertices. Let 0 denote the origin in $E^{3}$. Then it is straightforward to verify that 


$$
\left[0, g_{i}(0), g_{i} g_{j}(0), g_{i} g_{j} g_{k}(0)\right]
$$

are nondegenerate simplices in $E^{3}$, where $i, j, k$ form a permutation of $1,2,3$. Let $F$ denote the union of the above sets under all the permutations of $1,2,3$. It will now be our object to prove that $F$ is a fundamental domain for $\Pi_{3}^{3}$.

Let $F(2)=\left[0, g_{1}(0), g_{1} g_{3}(0)\right]\left[0, g_{3}(0) ; g_{1} g_{3}(0)\right]$ and let $R_{0}(2)$ $=\bigcup g_{1}^{n} g_{3}^{s} F(2)$ for all integers $n$ and $s$. By cyclic permutation we may define $F(i)$ and $R_{0}(i), i=1,2,3$.

Leмma 3. The projection $p$ of $R_{0}(2)$ perpendicular to the plane $z=0$ is a one-to-one mapping of $R_{0}(2)$ onto the plane $z=0$.

Proof. The perpendicular projection of a two-simplex onto a plane is one-to-one unless the image of the simplex is a line. Now the vertices of $R_{0}(2)$ map into the points with coordinates $(\lambda(n, s, m=0)$, $n+s q, 0)$. Consider the group $\Pi^{1}(2)$ of transformations of the plane $z=0$ generated by $\bar{g}_{1}$ and $\bar{g}_{3}$, where $\bar{g}_{1}$ and $\bar{g}_{3}$ are given by

$$
\begin{aligned}
x^{\prime}=x+y, & x^{\prime}=x+q y+k, \\
y^{\prime}=y+1, & y^{\prime}=y+q,
\end{aligned}
$$

respectively. Now $p\left(g_{1}^{n} g_{3}^{s}(0)\right)=\bar{g}_{1}^{n} \bar{g}_{3}^{s}(0)$. Hence the range of $g_{1}^{n} g_{3}^{s}[0$, $\left.g_{i}(0), g_{i} g_{j}(0)\right]$ under $p$ is $\bar{g}_{1} \bar{g}_{3}^{s}\left[0, \bar{g}_{i}(0), \bar{g}_{i} \bar{g}_{j}(0)\right]$, where $i, j$ form a permutation of 1 and 2 . Since the plane $z=0$ under the action of $\Pi^{1}(2)$ is a torus we must have that $\bar{g}_{1}^{n} \bar{g}_{3}^{s}\left[0, \bar{g}_{i}(0), \bar{g}_{i} \bar{g}_{j}(0)\right]$ are never degenerate. Hence $p$ is a one-to-one mapping of each of the simplices of $R_{0}(2)$. Further $p$ must be one-to-one in the large since $\left[0, \bar{g}_{1}(0), \bar{g}_{1} \bar{g}_{3}(0)\right]$ $\cup\left[0, \bar{g}_{3}(0), \bar{g}_{1} \bar{g}_{3}(0)\right]$ is a fundamental domain for the plane $z=0$ under the action of $\Pi^{1}(2)$. This also implies that the mapping $p$ is onto.

We have also shown

LEMMA 4. If $\Pi(2)$ is the group generated by $g_{1}$ and $g_{3}$ then $R_{0}(2) / \Pi(2)$ is homeomorphic to a two-dimensional torus and $F(2)$ is a fundamental domain for $\Pi(2)$.

Let $R_{\boldsymbol{s}}(i)=g_{i}^{s} R_{0}(i)$.

LEMMA 5. $R_{0}(2) \cap R_{1}(2)=0$.

Proof. Let us assume this lemma is false. Then there exist points $f_{1}$ and $f_{2} \in F(2)$ such that $g_{1}^{n} g_{3}^{s} f_{1}=g_{2} g_{1}^{n^{\prime}} g_{3}^{s^{\prime}} f_{2}$. Hence $g_{2} f_{2}=g^{n-n^{\prime}} g_{3}^{s-s^{\prime}} f_{1}$. But it is trivial to see that the $p\left[g_{2}(F(2))\right]$ meets $R_{0}(2)$. This contradicts Lemma 3 unless $n=n^{\prime}$ and $s=s^{\prime}$. But then $g_{2} f_{1}=f_{2}$ or $g_{2} F(2)$ $\cap F(2)$ is not empty. But this is false. 
Lemma 6. $R_{m}(2)$ separates $E^{3}$ and $R_{m}(2)$ lies between $R_{m-1}(2)$ and $R_{m+1}(2)$ for all $m$.

This follows from Lemmas 3 and 5 and the fact that $\Pi_{3}^{3}$ is orientation preserving.

LEMmA 7. Let $f_{1}$ and $f_{2}$ be between $R_{0}(2)$ and $R_{1}(2)$. If $g \in \Pi_{3}^{3}$ exists such that $g\left(f_{1}\right)=f_{2}$ then $g \in \Pi^{1}(2)$.

This is a trivial consequence of Lemma 6.

Now we may prove Lemmas 3 through 7 replacing 2 by 1 and 3 . Hence we have proved that $F$ is a fundamental domain for $\Pi_{3}^{3}$.

TheOREM. $E^{3} / \Pi_{3}^{3}$ is a torus.

This follows from the fact that $F$ is the fundamental domain and the identifications on the boundary of $F$.

\section{REFERENCES}

1. L. Auslander and L. Markus, Holonomy of flat affinely connected manifolds, Ann. of Math. vol. 62 (1955) pp. 139-151.

2. N. H. Kuiper, Sur les surfaces localement affines. Geométrie Differentielle, Colloques Internationaux du Centre National de la Recherche Scientifique, Strasbourg, 1953, pp. 79-87, Paris, Centre National de la Recherche Scientifique, 1953.

YALE UNIVERSITY 